
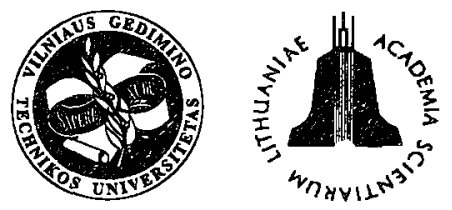

ISSN 1648-4142 TRANSPORT

http:/www.vtu.lt/english/editions

TRANSPORT - 2003, Vol XVIII, No 1, 49-55

\title{
DYNAMIC PROCESSES IN THE OIL PIPELINES. PART II. THE LEAK IDENTIFICATION BY NUMERICAL METHODS
}

\author{
Ingrida Bajoraitytė ${ }^{1}$, Marijonas Bogdevičius ${ }^{2}$ \\ Dept of Transport Technological Equipment, Vilnius Gediminas Technical University, \\ Plytines g. 27, LT - 2016 Vilnius, Lithuania. Tel. (5) 2744782
}

Received 20020701 ; accepted 20021221

\begin{abstract}
The analysis of identification method of leaks occurring due to damages of the linear part of the main oil pipelines is carried out. The momentum and continuity equations of viscous compressible fluid in a pressure pipeline are presented. Differential equations of fluid movement in the oil pipeline are solved by the method of characteristics. Variation diagrams of pressure and velocity of fluid of a leaking oil pipeline are presented.
\end{abstract}

Keywords: oil pipeline; damage; identification; numerical method; wave speed.

\section{Introduction}

The classification of methods of identification of places of outflow of a liquid in the main pipelines are submitted in works [1-3]. One of them has merits and demerits.

For fast detection of places of outflow of liquid in the main pipelines the most effective is the method of impact wave which takes into account waves of pressure in liquid in the main pipelines

On the basis of this method the method and the software of identification of places of outflow of liquid in the main pipelines is developed.

Since the proportion of the fluid transported through these connections is rather small, the identification of leaks is very complicated.

\section{Mathematical Model of Pipeline}

The flow of viscous and compressive fluid is described by differential equations with partial derivatives, which express the laws of mass movement, quantity and conservation of energy. The equation of fluid condition is added to differential equations, which relate thermodynamic variables. These equations with initial and boundary conditions influence on the solution and can be essentially integrated during time. Therefore, fluid flow parameters at certain moments can be estimated.

When solving complicated applied hydrodynamic tasks non-stationary quasi-linear one-dimensional fluid flow models are widely applied [1-16]. In such models the condition of flow at every moment of time is charac-

\footnotetext{
${ }^{1}$ E-mail: marius@ti.vtu.lt

${ }^{2}$ E-mail: inga@post.omnitel.net
}

terized by average values of pressure, velocity and density. When estimating the average values of fluid flow parameters the following pre-conditions are taken into consideration: pressure, density, internal energy, and temperature change inconsiderably in a cross-section of a pipeline; the speed of movement on the walls of a pipeline is equal to zero.

We will study one-dimensional non-stationary isothermic movement of viscous and compressive fluid in a pipeline; i.e. when the fluid velocity vector is directed longitudinally to the axis of a pipeline, and speed and pressure change during time and longitudinally to the axis of a pipeline (coordinate $x$ ).

Movement and continuity equations of viscous, compressible fluid in a pressure pipe have the following form:

$$
\begin{aligned}
& \frac{1}{\rho} \frac{\partial p}{\partial x}+\frac{\partial v}{\partial t}+v \frac{\partial v}{\partial x}+\frac{f v v}{2 d}+a_{x}=0 \\
& \frac{\partial p}{\partial t}+v \frac{\partial p}{\partial x}+c^{2} \rho \frac{\partial v}{\partial x}=0
\end{aligned}
$$

here: $p, v-$ pressure and velocity of fluid; $\rho$ - fluid density; $c$-velocity of sound; $f$-non-dimensional hydraulic coefficient of friction.

Differential equations of fluid movement in the cylinder are solved by the method of characteristics [1, 2]. The essence of the method of characteristics is that the speed of unknown variable and fluid pressure at instant moment of time $t+\Delta t$ are determined according to these parameters at a moment of time (Fig 1).

Pressure and velocity in point $D$ at the moment of time is determined from nonlinear algebraic equation system 


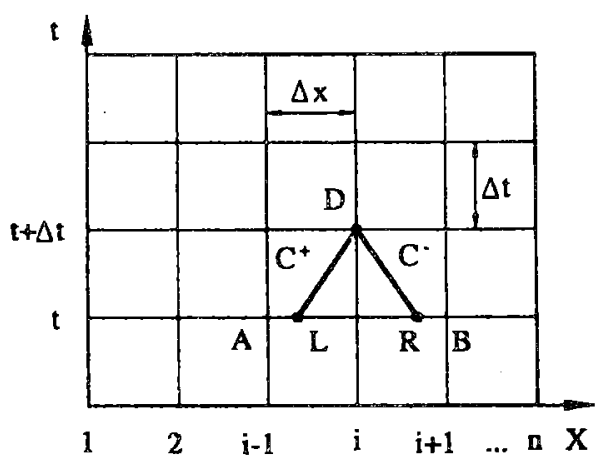

Fig 1. The circuit of point parameters determination by the method of characteristics

$C^{+}: \Phi_{1}=v_{D}-v_{L}+\frac{1}{2}\left(p_{D}-p_{L}\right)\left[\left(\frac{1}{\rho c}\right)_{L}+\left(\frac{1}{\rho c}\right)_{D}\right]-$

$\frac{\Delta t}{2}\left[\left(\frac{f_{1}}{\rho c}\right)_{L}+\left(\frac{f_{1}}{\rho c}\right)_{D}\right]-\frac{\Delta t}{2}\left[\left(f_{2}\right)_{L}+\left(f_{2}\right)_{D}\right]=0$

$C^{-}: \Phi_{2}=v_{D}-v_{R}-\frac{1}{2}\left(p_{D}-p_{R}\right)\left[\left(\frac{1}{\rho c}\right)_{R}+\left(\frac{1}{\rho c}\right)_{D}\right]+$

$\frac{\Delta t}{2}\left[\left(\frac{f_{1}}{\rho c}\right)_{R}+\left(\frac{f_{1}}{\rho c}\right)_{D}\right]-\frac{\Delta t}{2}\left[\left(f_{2}\right)_{R}+\left(f_{2}\right)_{D}\right]=0 ;$

$$
f_{1}(v, p)=\frac{c_{2}}{S(x)}\left(F_{1}-\rho \nu \frac{\partial S}{\partial x}\right)
$$

$f_{2}(v, p)=\frac{1}{\rho S(x)}\left(F_{2}-v F_{1}\right)-\frac{\tau \Pi(x)}{\rho S(x)}-a_{x}$

$F_{1}=\Pi(x) m_{\text {inp }}(x) v_{\text {inp }}(x) ; F_{2}=\Pi(x) n_{\text {inp }}(x) v_{\text {inp }}(x)$;

$$
\tau=\frac{\rho f(\operatorname{Re}) v i v !}{8}
$$

$f(\operatorname{Re})=\left\{\begin{array}{l}\frac{75}{\operatorname{Re}}, \text { when } \quad \operatorname{Re} \leq 2320 ; \\ \frac{0,31464}{\operatorname{Re}^{0,25}}, \text { when } \quad \operatorname{Re}>2320 .\end{array}\right.$

The velocity of sound in a two-phase fluid in a pipeline is as follows:

$$
c=\sqrt{\frac{K(p) / p}{1+\frac{K(p) \cdot d}{E \cdot e}+\frac{\varepsilon}{\gamma}\left[\frac{K(p)}{\gamma p}-1\right]},}
$$

here $K(p)$ - volumetric elasticity modulus of fluid, $\rho-$ fluid density, $E$ - elasticity modulus of a pipeline, $d$ internal diameter of a pipeline, $e$-thickness of a pipeline wall, $\gamma$-index of adiabatic process, $\varepsilon$ - rate of gas volume in fluid with the total volume of fluid (mixture).

In general, volumetric elasticity modulus depends on pressure:

$$
K(p)=\frac{\sum_{i=0}^{n_{1}} a_{i} p^{i}}{\sum_{j=0}^{n_{2}} b_{i} p^{j}}
$$

here: $a_{i}, b_{i}$-coefficients which are identified by experiments. In general, the sound velocity in medium is equal to.

Non-linear system of algebraic equations (4) and (5) is solved by Newton method.

To enable the stability of solution, Currant's condition shall be taken into account:

$$
\Delta t|v+a| \leq \Delta x .
$$

Characteristics $C^{+}$passes through points $L$ and $D$, the equation of which is:

$$
\frac{d x}{d t}=v+a .
$$

Having integrated expression between points $L$ and $D$, we receive coordinate $\mathrm{x}$ of point $L$ :

$$
x_{L}=x_{D}-\left(v_{L}+a_{L}\right) \Delta t
$$

Velocity and pressure are approximated by the first degree polynomials between points $A$ and $C$ :

$$
\begin{aligned}
& v(x)=\frac{x_{D}-x}{\Delta x} v_{A}+\frac{x-x_{A}}{\Delta x} v_{c}, \\
& p(x)=\frac{x_{D}-x}{\Delta x} p_{A}+\frac{x-x_{A}}{\Delta x} p_{c} .
\end{aligned}
$$

Having written expression (12) in expressions (10), we receive the system of two non-linear algebraic equations with unknown quantities $p_{L}$ and $v_{L}$ :

$$
\begin{aligned}
& \Phi_{3}=p_{L}-p_{C}-\theta\left(p_{A}-p_{C}\right)\left[v_{L}+a\left(p_{L}\right)\right\rfloor=0, \\
& \Phi_{4}=v_{L}-v_{C}-\theta\left(v_{A}-v_{C}\right)\left[v_{L}+a\left(p_{L}\right)\right\rfloor=0,
\end{aligned}
$$

here: $\theta=\Delta t / \Delta x$.

The system of non-linear algebraic equations (13) and (14) is solved by Newton method.

The values of variables at point $R$ are estimated analogously. In this case we receive the system of two non-linear algebraic equations with unknown quantities $p_{R}$ and $v_{R}$ :

$\Phi_{5}=p_{R}-p_{c}+\theta\left(p_{B}-p_{c}\right)\left\lfloor v_{R}-a\left(p_{R}\right)\right\rfloor=0$, 


$$
\Phi_{6}=v_{R}-v_{c}+\theta\left(v_{B}-v_{c}\right)\left[v_{R}-a\left(p_{R}\right)\right]=0
$$

The system of non-linear algebraic equations (15) and (16) also is solved by Newton method.

The system of equations (3) and - (4) is solved by Newton method:

$$
\begin{aligned}
& \lfloor J\rfloor_{j}\{Y\}_{j}=-\{\Phi\}_{j}, \\
& \{Y\}=\left[p_{D}, v_{D}\right\}\{\Phi\}=\left[\Phi_{1}, \Phi_{2}\right] .
\end{aligned}
$$

The nodal point of three pipes is presented in (Fig 2).

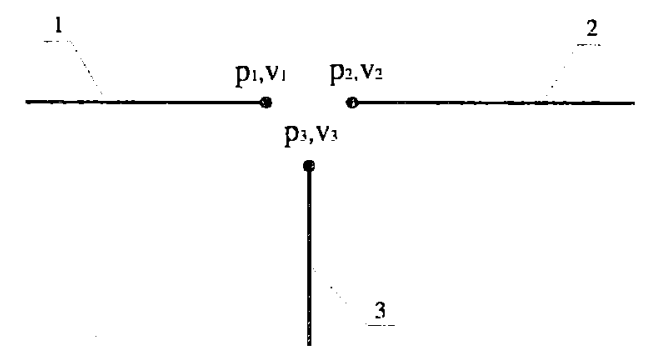

Fig 2. The circuit of three connected pipes: 1 - first pipe,

$$
2 \text { - second pipe, } 3 \text { - third pipe }
$$

Pressure and velocity in a nodal point are as follows: on last nodal point of the first pipe $-v_{1}$ and $p_{1}$, on the first nodal point of the second pipe $-v_{2}$ and $p_{2}$, on the first nodal point of the third pipe $-v_{3}$ and $p_{3}$. Five unknown quantities are received in the common nodal point: $p_{1}=p_{2}=p, p_{3}, v_{1}, v_{2}$ and $v_{3}$. To estimate the unknown, the system of five equations shall be identified. According to formulae (3) and (4), $C^{+}$and $C^{-}$characteristics are written:

$$
\begin{aligned}
& C^{+}: \Phi_{1}=\Phi_{1}\left(p_{1}, v_{1}\right) ; \\
& C^{+}: \Phi_{2}=\Phi_{2}\left(p_{2}, v_{2}\right) ; \\
& C^{-}: \Phi_{13}=\Phi_{3}\left(p_{3}, v_{3}\right) .
\end{aligned}
$$

The fourth equation in the common nodal point is written on the following condition:

$$
\sum_{i=1}^{3} Q^{(i)}=0
$$

here: $Q^{(i)}=S_{i} v_{i}$ - fluid capacity in the nodal point ("+" - fluid flows into nodal point, "-"-fluid flows from nodal point), $S_{i}$-cross-section area of pipe $i, v_{i}$-velocity of fluid in pipe $i$.

The fourth equation is as follows:

$$
\Phi_{4}=S_{1} v_{1}-S_{2} v_{2}-S_{3} v_{3}=0
$$

The fifth equation is as follows:

$$
\Phi_{5}=p-p_{3}-\frac{1}{2} \xi \rho\left(\nu_{3}\right)^{2} \operatorname{sign}\left(\nu_{3}\right)=0,
$$

here: $\xi$ - coefficient of local losses which depends on the rate of pipe cross-section area, $\rho$ - fluid density, signv-gives an appropriate sign depending on the speed.

The received system of five non-linear algebraic equations (18) - (23) is solved by Newton method [9]. Having solved it, the values of pressure $p_{1}=p_{2}=p$, $p_{3}$ and velocity $v_{1}, v_{2}, v_{3}$ in a nodal point are received.

\section{The Methodology of Leak Identification in a Pipe- line}

A crack in a pipeline causes rapid variations of fluid flow. The wave of rapidly reduced pressure propagates on both sides from the location of the crack at the speed of sound. When leakage occurs in a pipeline, pressure and velocity vary rapidly. Following the diagrams of pressure and velocity variations, fluid leak can be identified.

The method of leak identification is based on the observation of pressure wave propagation time when the change of pressure is registered. Identification of a leak is registered by the variation of time $\Delta t$ between pressure waves propagating into opposite sides from the leak which reach the beginning and the end of a pipeline, i.e. pressure sensing elements in the pipeline section under control, at different time. If leakage occurs in the middle of a pipeline section under control, variation of time $\Delta t$ will be equal to zero. Meanwhile, when leakage occurs closer to any end of the pipeline section under control, the variation of time $\Delta t$ of pressure wave propagation occurs [10].

A sample diagram of pressure variation is presented in (Fig 3).

In case of leakage, pressure falls rapidly. The starting point of pressure fall is identified with the first sensing element: pressure $p_{1}$ and time $t_{1}$ are identified on that point. The starting point of pressure fall is identified with the second sensing element: pressure $p_{2}$ and time $t_{2}$ are identified on that point. Time difference $\Delta t$ is calculated as well:

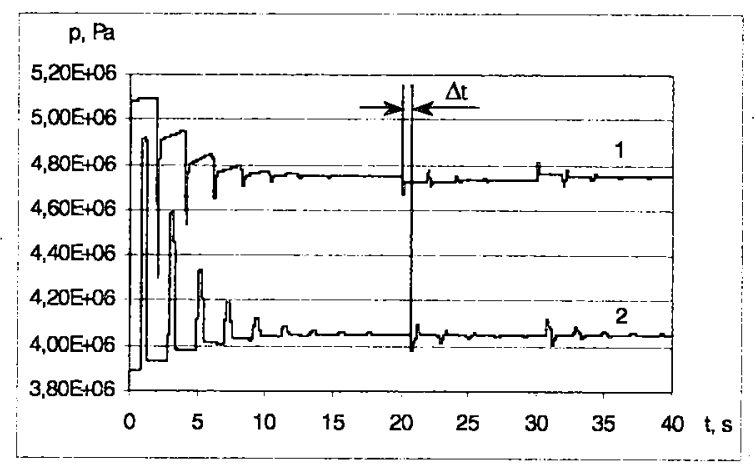

Fig 3. Registering of pressure variations in a pipeline when leakage occurs: 1 - data from the first sensing element, 2 data from the second sensing element 


$$
\Delta t=t_{2}-t_{1}
$$

here: $\Delta t-$ variation of time of waves propagating to the beginning and the end of the pipeline section under control, $t_{1}$ - time of wave propagation up to beginning of the pipeline section under control, $t_{2}$ - time of pressure wave propagation up to the end of the pipeline section under control.

Pressure pulses at the location of damage propagate at the speed of sound longitudinally to a pipeline (on both sides of cracking) and certain moments of time are as follows:

$$
t_{1}=\frac{x}{a}, t_{2}=\frac{L-x}{a},
$$

here:- distance from the beginning of the pipeline section under control up to the location of leakage, $L$ - length of pipeline section under control, $a$-sound propagation speed.

Variation of time when fluid pressure pulses reach the beginning and the end of a pipeline are equal to:

$$
\Delta t=t_{2}-t_{1}=\frac{l-2 x}{v} .
$$

Then the distance from the beginning of a pipeline section under control up to the leak is calculated according to the following formula:

$$
x=\frac{L}{2} \pm \frac{a}{2} \Delta t
$$

If the first sensing element reacts first, symbol "_" is written, i.e. leakage is closer to the beginning of a pipeline under control, but if the second sensing element reacts first, symbol " + " is written, i.e. leakage is closer to the end of a pipeline section under control.

Having taken the velocity of fluid flow into consideration when pressure wave propagation time $t_{1}$ and $t_{2}$ is:

$$
t_{1}=\frac{\frac{L}{2}-x}{a-v_{v i d}}, \quad t_{2}=\frac{\frac{L}{2}+x}{a+v_{v i d}}
$$

here: $v_{v i d}-$ fluid flow velocity.

The distance from the leak to the beginning of the pipeline section under control is calculated as follows:

$$
x=\frac{L}{2} \pm \frac{\Delta t\left(a+v_{\text {vid }}\right)\left(a-v_{\text {vid }}\right)+L v_{\text {vid }}}{2 a}
$$

Analogous calculations are carried out observing the variation of fluid flow velocity depending on time. In case of leakage, fluid flow registered by the first sensing element increases rapidly and the fluid flow velocity registered by the second sensing element starts falling rapidly. The starting point of fluid flow velocity increase is identified with the first sensing element: velocity $v_{1}$ and time $t_{1}$ are identified at that point. The starting point of fluid flow velocity fall registered by the second sensing element is identified as well: velocity $v_{2}$ and time $t_{2}$ are identified at that point as well. To identify leakage location analogous calculations are carried out.

A leak can be identified approximately for a concrete pipeline section having drawn the graph $\Delta t=f(x)$ of dependence. Air melted in fluid influences on the sound velocity propagation. This graphical dependence takes sound velocity variation longitudinally to the pipeline section into consideration. This dependence graph is drawn up according to the following formula [8]:

$$
\Delta t=\frac{L-x}{a_{1}+\frac{a_{1}-a_{2}}{2}\left(1-\frac{x}{L}\right)}-\frac{x}{a_{1}+\frac{a_{1}-a_{2}}{2}\left(2-\frac{x}{L}\right)}
$$

here: $a_{1}$ - sound speed at the beginning of a pipeline; $a_{2}$ - sound speed at the end of a pipeline.

\section{Findings of Research and their Analysis}

Three cases of pipelines are investigated in this research: pipeline sections of different length as well as different setting of sensing elements registering pressure in a pipeline.

The circuit of the pipeline section under investigation, setting of sensing elements registering pressure as well as distances of leaks are presented in Fig 4.

The total length of a pipeline section, $i$. e. distance between sensing elements, $L=750 \mathrm{~m}$; distance from the first leakage is $x_{1}=50 \mathrm{~m}$, and from the second is $x_{2}=550 \mathrm{~m}$.

Leakage in the system is simulated by closing and opening leaking pipes. Variations of velocity and pressure in the system were observed when the diameter of the first pipe $d_{1}$ is $5 \mathrm{~mm}, 15 \mathrm{~mm}, 50 \mathrm{~mm}, 100 \mathrm{~mm}$, and the opening time of simulated leakage is $t_{\text {open }}=20 \mathrm{~s}$ and closing time by simulating the end of leakage is $t_{\text {close }}=30 \mathrm{~s}$. The diameter of the second pipe $d_{2}$ is $10 \mathrm{~mm}, 20 \mathrm{~mm}, 75 \mathrm{~mm}$, and the opening time when simulating leakage is $t_{\text {open }}=40 \mathrm{~s}$ and the closing time when

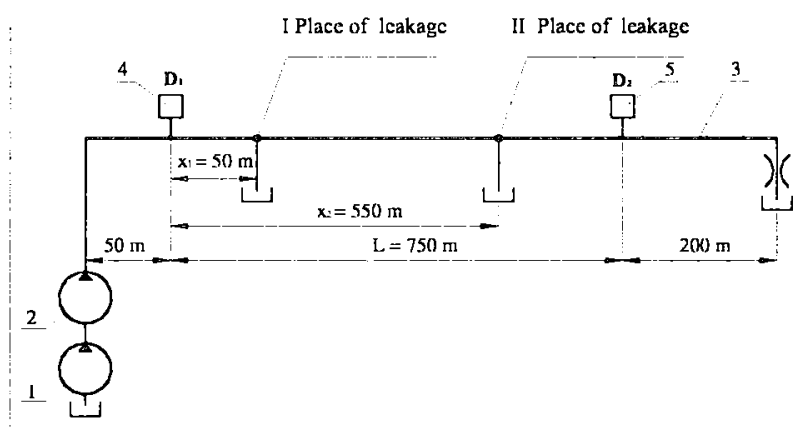

Fig 4. Circuit of $1 \mathrm{~km}$ long pipeline section: 1,2-consistently coupled centrifugal pumps of mark HM $5000-210$; 3 - pilot pipeline section; 4,5- sensing elements which register pressure 
simulating the end of leakage is $t_{\text {open }}=50 \mathrm{~s}$.

Two centrifugal pumps of mark HM 5000-210 are consistently mounted at the beginning of a pipeline. Characteristics of a pump are defined by the developed pressure dependence $H$ on input $Q$. Characteristics of centrifugal pumps used in the main oil pipelines are fluently falling curves.

Characteristics of consistently coupled pumps of mark HM 5000-210 are presented in Fig 5.

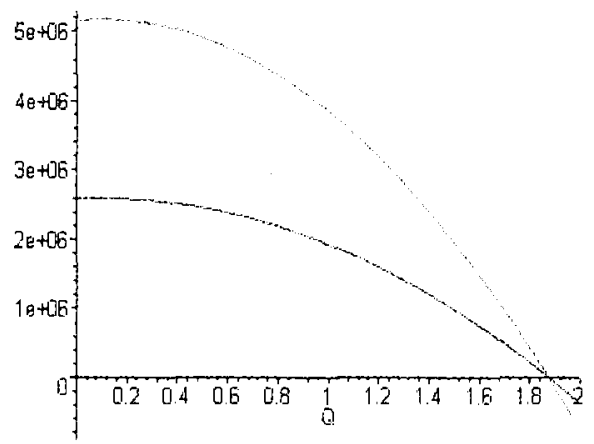

Fig 5. Characteristics of consistently connected pumps of mark HM $5000-210$

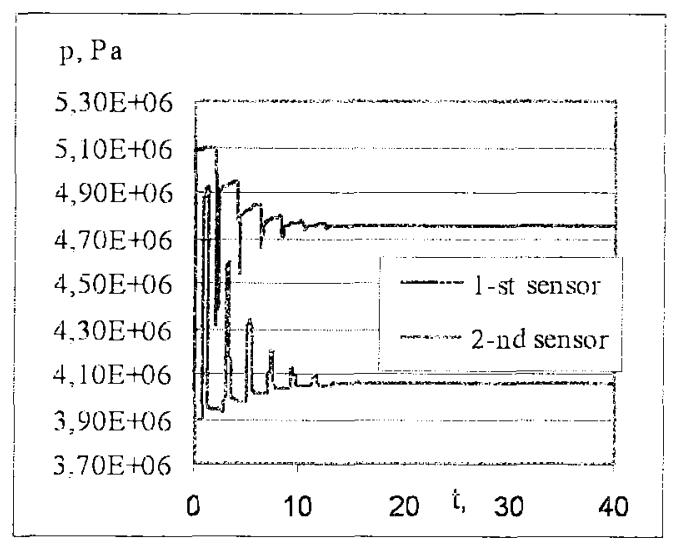

Fig 6. Pressure variation diagram when leakage is absent in a pipeline

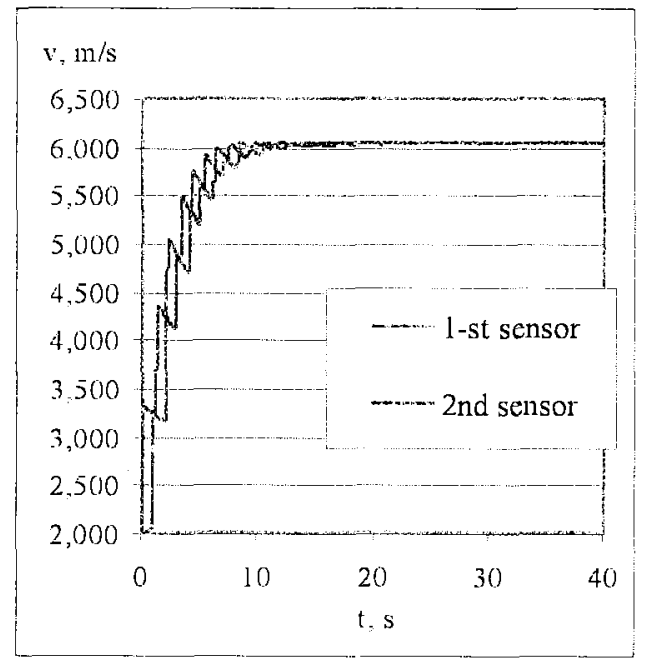

Fig 7. Velocity variation diagram when leakage is absent in a pipeline
Diagrams of pressure and velocity variation when the leakage is absent in the system are presented in Fig 6 and Fig 7.

Diagrams of pressure and velocity variation at different diameters of leaking pipe and different distances of leaks from the beginning of a pipeline section under control are presented in Fig 8-15.

Data and calculation results when the leak is identified according to the variations of velocity when diameters of leaking pipes are different (the length of a pipeline section is $1 \mathrm{~km})[16,17]$.

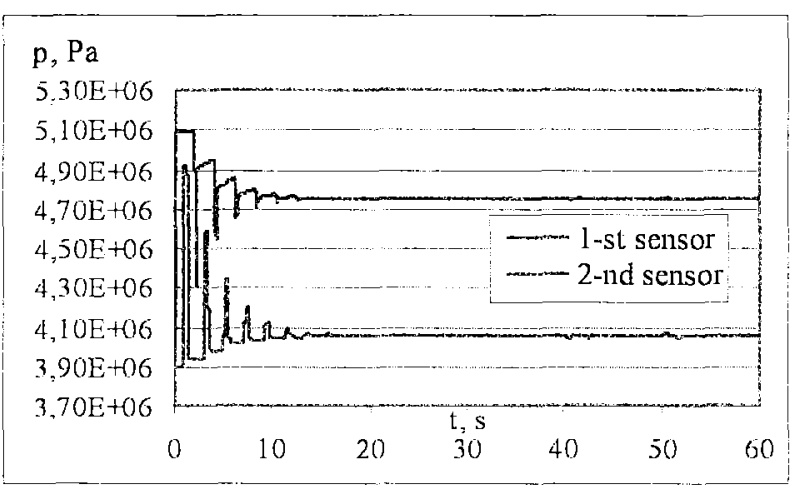

Fig 8. Pressure variation diagram when the diameter of the first leaking pipe is $5 \mathrm{~mm}$, and the second $-10 \mathrm{~mm}$

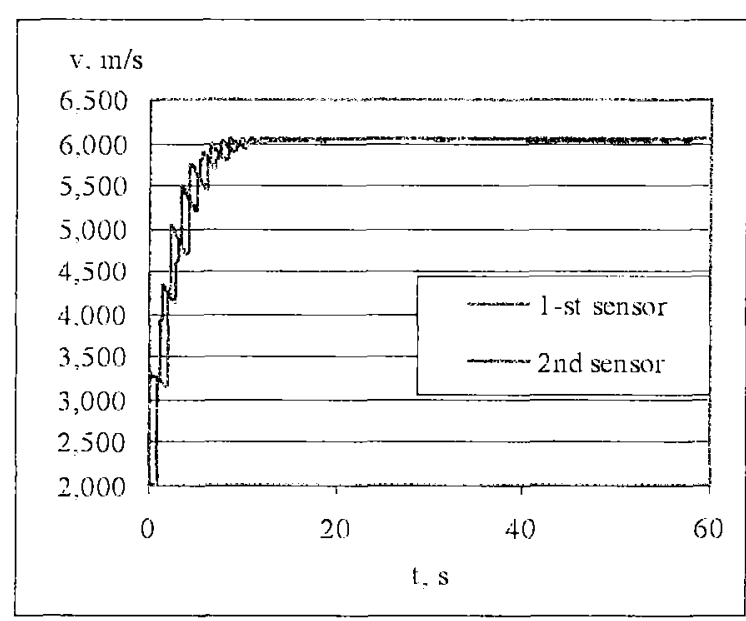

Fig 9. Velocity variation diagram when the diameter of the first leaking pipe is $5 \mathrm{~mm}$, and the second $-10 \mathrm{~mm}$

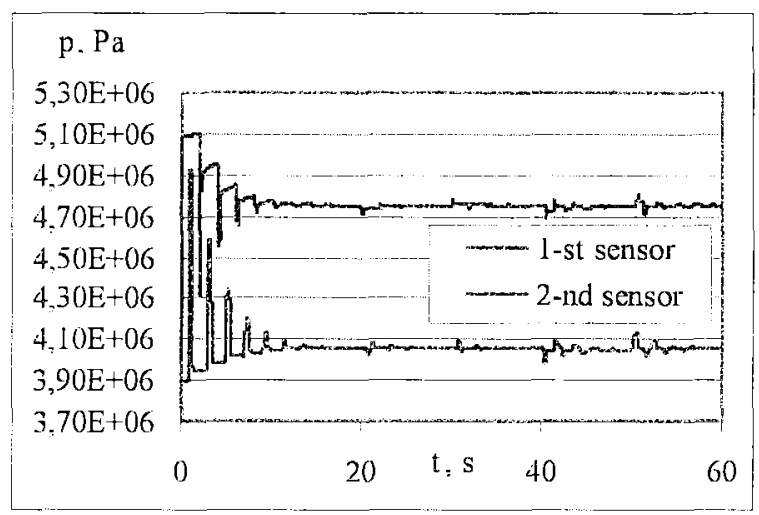

Fig 10. Pressure fall diagram when the diameter of the first leaking pipe is $15 \mathrm{~mm}$, and the second $-20 \mathrm{~mm}$ 


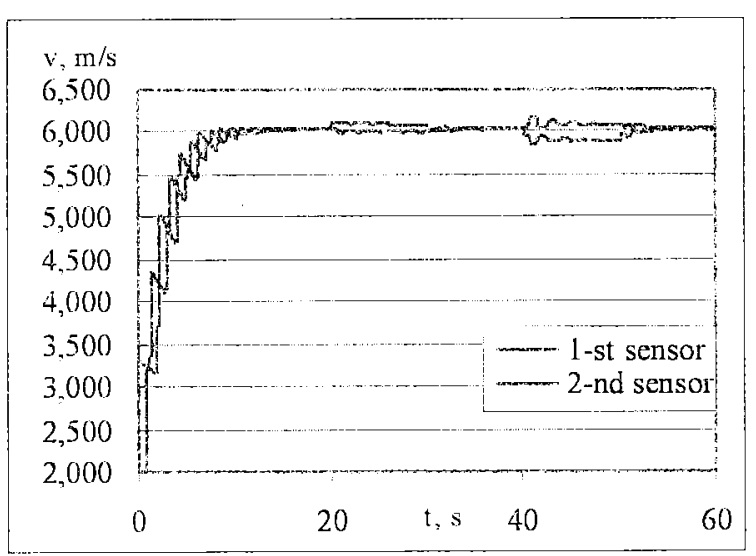

Fig 11. Velocity variation diagram when the diameter of the first leaking pipe is $15 \mathrm{~mm}$, and the second $-20 \mathrm{~mm}$

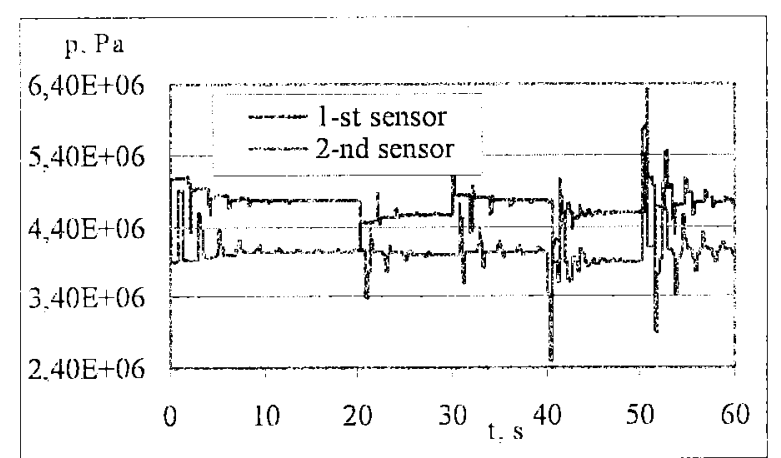

Fig 12. Pressure variation diagram when the diameter of the first leaking pipe is $50 \mathrm{~mm}$, and the second $-75 \mathrm{~mm}$

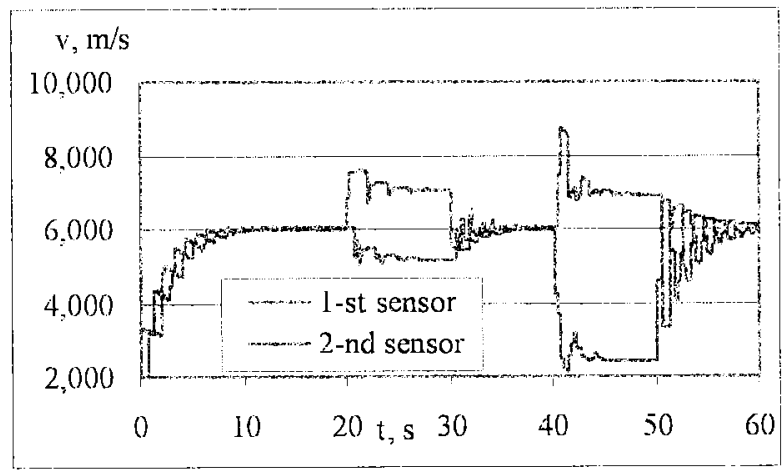

Fig 13. Velocity variation diagram when the diameter of the first leaking pipe is $50 \mathrm{~mm}$, and the second $-75 \mathrm{~mm}$

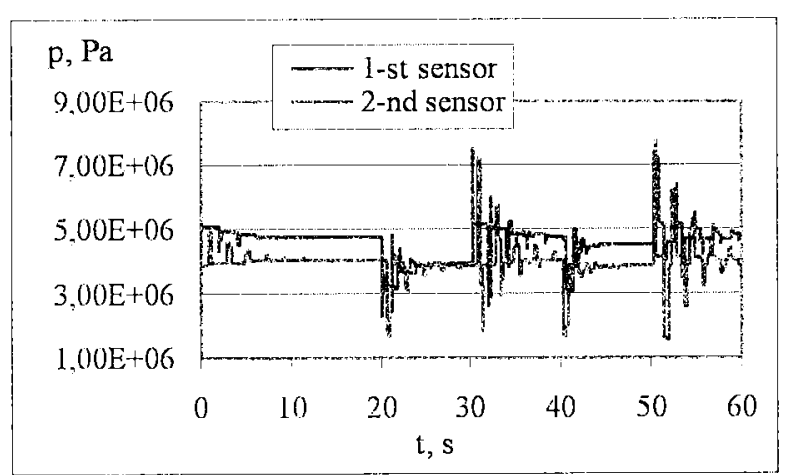

Fig 14. Pressure variation diagram when the diameter of the first leaking pipe is $100 \mathrm{~mm}$, and the second $-100 \mathrm{~mm}$

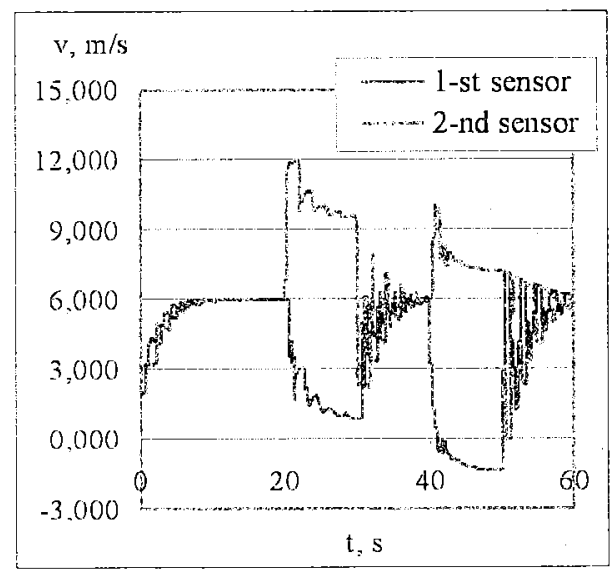

Fig 15. Velocity variation diagram, when the diameter of the first leaking pipe is $100 \mathrm{~mm}$, and the second $-100 \mathrm{~mm}$

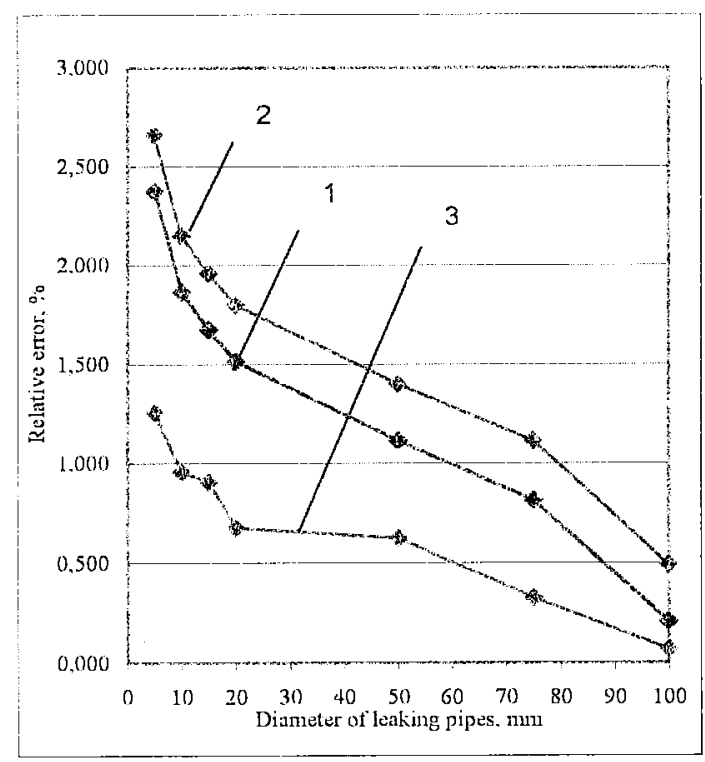

Fig 16. Dependences of relative errors on the diameter of a leaking pipe when the length of a pipeline section is $1 \mathrm{~km}$ : 1 - calculation on pressure and taking into account velocity of liquid; 2 - calculation on pressure and not taking into account velocity of liquid; 3 - calculation on liquid

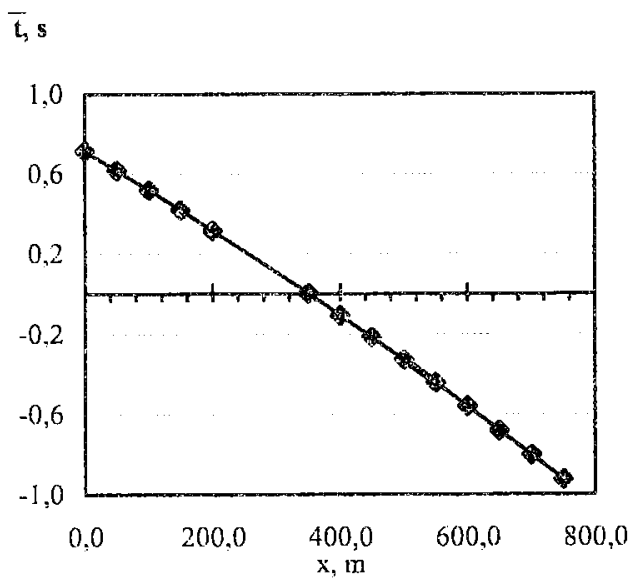

Fig 17. Graph of dependence $\Delta t=f(x)$ for $1 \mathrm{~km}$ long pipeline section 


\section{Conclusions}

1. When analyzing pressure and velocity variation diagrams, on the pipeline section under control at various leakage holes diameter it was identified that the larger the diameter of a leakage hole is, the greater variations of pressure and fluid flow velocity in a pipeline at leakage are.

2. The distance from the beginning of the pipeline section under control to the leak is identified much more accurately when the diamater of a leaking hole is greater. When the diameter of a leakage hole is $10 \mathrm{~mm}$, the relative error of leak estimation is $3.575 \%$, and when the diameter of a leakage hole is $100 \mathrm{~mm}$, the relative error of the identified leak is $1,627 \%$.

3. It was identified that the distance from the beginning of a pipeline section under control to the leakage location is calculated more accurately when calculations are carried out according to the variations of fluid flow velocity than calculating according to the pressure variations in the system. When the diameter of a leakage hole is $10 \mathrm{~mm}$, a relative error of leak estimation is $3,575 \%$ if calculated according to the pressure variation, a relative error of leak estimation is 3,190\% if calculated according to the velocity variation. Therefore, it is recommended to estimate the leak in a pipeline according to the variations of fluid flow velocity but not according to pressure variations.

\section{References}

1. Alijev, R. Pipeline Transport of Oil and Gas (Tpyбoпроводный транспорт нефти и газа). Moscow, 1988. 368 p. (in Russian).

2. Perfecting of Acoustic and Emission Search of Leakages in Pipelines. In: Oil and Gas Industry (Совершенствование акустоэмиссионного поиска утечек в трубопроводах). Moscow, 1986, No 2, p. 51 - 52 (in Russian).

3. Bogdevicius, $M$. Identification of pipeline and fluid parameters by the method of characteristics. Transport Engineering (Transportas), Vol XV, No 3, 2000, p. $150-152$ (in Russian).

4. Bajoraitytè, I.; Bogdevičius, M. Dynamic Processes in the Oil pipelines. Part I. The analysis of causes of leakage occurrence in oil pipelines and Leak identification methods. Transport, Vol XVII, No 6. 2002, p. 234-240.

5. Agapkin, B. The Final Analysis of Science and Engineering. A Pipeline Transport (Итоги науки и техники. Трубопроводньй транспорт). Moscow, 1984. 105 p. (in Russian).

6. Below, N. The Analysis of Accident Rates on Gas and Oil Pipelines (Анализ аварийности на газонефтепроводах). Moscow, 1990. 319 p. (in Russian).

7. Bogdevicius, $M$. Non-stationary movement of fluid in the elastic, visco-elastic, and plastic pipe. Applied Mechanics (Taikomoji mechanika), No 2, 1994, p. 117 - 124 (in Lithuanian).
8. Aladjev, V.; Bogdevicius, M.; Prentkovskis O. New Software for Mathematical Package Maple of Releases 6, 7 and 8. Vilnius: Technika, 2002. 403 p.

9. Aladjev, V.; Bogdevicius, M. Maple 6: Solution of the Mathematical, Statistical and Engineering-physical Problems (Maple 6: Решение математических, статистических и инженерно-физических задач). Moscow, 2001. 824 р. (in Russian).

10. Bogdevičius, M. Simulation Interaction of Mechanical and Hydraulic System. In: Tenth World Congress on the Theory of Machines and Mechanisms. Oulu, Finland, 1999, p. 2110 -2115 .

11. Bogdevičius, M. Simulation of Hydraulic Systems of the Pump by the Method of Characteristics. Transport Engineering (Transportas), 1997, No 2(15), p. $30-37$ (in Russian).

12. Borodavkin, P. Underground Main Pipelines (Подземные магистрапьные трубопроводы). Moscow, 1982. 384 p. (in Russian).

13. Borisov, V. Control of the Main Pipelines (Управление магистральными трубопроводами). Moscow, 1979. 215 p. (in Russian).

14. Pipeline Transport. The Final Analysis of Science and Engineering, Volume 3 (Трубопроводный транспорт. Итоги науки и техники, том 3). Moscow, 1990. 120 p. (in Russian).

15. Non-destructive Inspection and Diagnostics of Pipelines (Неразрушаюший контроль и диагностика трубопроводов). Moscow, 1988. 159 p. (in Russian).

16. Michael, J. Here are the factors that govern evaluation of mechanical damage to pipelines. Oil \& Gas Journal, Sept. 9, 2002, p. 64-69. 